\title{
ROLE OF VASP PHOSPHORYLATION ASSAY IN MONITORING THE ANTIPLATELET THERAPY
}

\author{
Fedor M. ${ }^{1}$, Simonova R. ${ }^{1}$, Fedorova J.. ${ }^{2}$, Skornova I. ${ }^{1}$, Duraj L. ${ }^{1}$, Samos M ${ }^{3}$, \\ Stasko J. ${ }^{1}$, Kovar F. ${ }^{3}$, Mokan M. ${ }^{3}$, Kubisz P. ${ }^{11}$
}

\begin{abstract}
National Center of Thrombosis and Haemostasis, Clinic of Haematology and Transfusiology, Jessenius Faculty of Medicine and University Hospital, Martin, Slovak Republic

${ }^{2}$ Hemo-Medika s.r.o., Center of Haemostasis and Thrombosis, Martin

${ }^{3} 1$ st Clinic of Internal Medicine, Jessenius Faculty of Medicine and University Hospital, Martin, Slovak Republic
\end{abstract}

\begin{abstract}
Dual antiplatelet treatment with clopidogrel and aspirin represents standard regimen in prevention of thromboembolic events in patients with ischemic heart disease undergoing percutaneous coronary intervention (PCI). One of the greatest pitfalls of clopidogrel treatment is large inter-individual variability in response. Large amount of patients does not respond adequately and therefore are not „protected“ even in spite of receiving the therapy. Poor responders are exposed to three-fold increased risk of myocardial infarction, stent thrombosis and cardiac death.

Clopidogrel is an antiplatelet prodrug, whose active metabolite inhibits platelet function by irreversible binding to the (adenosine diphosphate) platelet receptor P2Y12. Receptor P2Y12 plays primal role in ADP-mediated platelet activation, and also in mechanism of action of ADP inhibitors such as clopidogrel, prasugrel etc. Reasons stated above, raised the necessity for implementing reliable laboratory test in order to identify the unprotected patients. In an ideal scenario, such test would serve to adjust the dose and guide the individual tailored treatment.

Vasodilator Stimulated Phosphoprotein (VASP) is an intracellular platelet protein which is non phosphorylated at basal state. Since its relation in cascade with P2Y12 receptor, VASP phosphorylation corerlates with inhibition of $\mathrm{P} 2 \mathrm{Y} 12$ which is the receptor of prime importance in ADP mediated activation of platelets and as is primary target of ADP inhibitors action. Outcome of the assay is represented as the value of platelet reactivity index (PRI), where PRI values above $50 \%$ are considered inadequate response to treatment and signal exposure to increased risk of myocardial infarction, post-PCI stent thrombosis and cardiac death. VASP-P flow cytometric assay is emerging into the spotlight as the promising method, mostly for its specificity for ADP inhibitors, better outlook for standardising results and lesser sample manipulation compared to multiple electrode aggregometry.
\end{abstract}

Keywords : acute coronary syndrome, clopidogrel, flow cytometry, platelet reactivity, vasodilator stimulated phosphoprotein (VASP)

\section{ROLE OF P2Y RECEPTORS IN PLATELET ACTIVATION}

Proaggregatory effect of ADP is mediated through interaction between ADP and G-protein coupled thrombocyte surface receptors : P2Y1 and P2Y12. Following its activation by ADP , receptor $\mathrm{P}_{2} \mathrm{Y}_{1}$ triggers change in thrombocyte shape and mediates weak transient aggregation. However, it is receptor P2Y12 whose activation is necessary for complete platelet response to ADP, as well as ADP-mediated response to other inductors (such as thrombin, TXA2, collagen etc.). P2Y12 also maintains prominent role in stimulating platelet secretion (platelet degranulation and production of thrombin) and activation of GPIIb/IIIa which binds fibrinogen and links platelets. Its presence is limited to platelets, endothelial cells and smooth muscle cells. This fact along with receptors" importance in platelet activation makes P2Y12 an attractive target for effective prevention of thromboembolism [1].

Address for correspondence:

Marian Fedor, MD, Clinic of Haematology and Transfusiology, JFM CU, University Hospital, Kollarova Str. N. 2, 03601 Martin, Slovak Republic; e-mail: duraj@jfmed.uniba.sk 


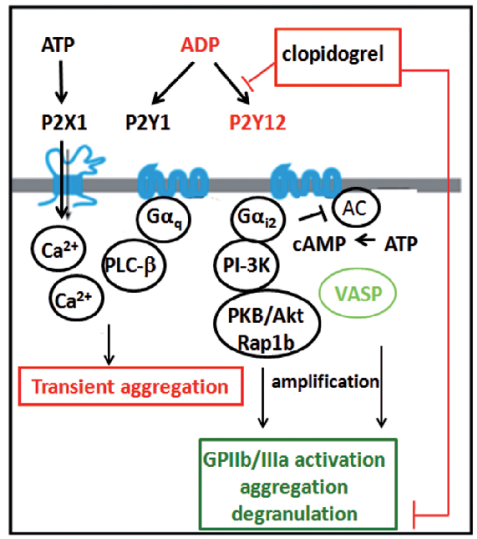

Fig. 1. Thrombocyte P2Y receptors and their role in ADP mediated platelet activation (Adapted from: Cattaneo M.et al, Aspirin and Clopidogrel : Efficacy, Safety, and the Issue of Drug Resistance ,Arteriosclerosis, Thrombosis, and Vascular Biology. 2004; 24: 1980-1987; Montalescot G, Wiviott SD, Prasugrel compared with clopidogrel in patients undergoing percutaneous coronary intervention for ST-elevation myocardial infarction (TRITON-TIMI 38): doubleblind, randomised controlled trial. Lancet 2009; 373: 723-731) [2,3].

\section{CLOPIDOGREL}

Mechanism of action of thienopyridine derivatives such as ticlopidine and clopidogrel is based on binding to platelet ADP receptor. Thienopyridine compounds are prodrugs and need to be metabolized in liver (CYP450) to attain their therapeutic effect. Clopidogrel has widely replaced ticlopidine due to its better safety and tolerability profile. The combination of clopidogrel and aspirin represents the mainstay in the prevention of major adverse cardiovascular events (MACEs) in patients with acute coronary syndromes $[4,5]$.

Clopidogrel is a prodrug, that first needs to be metabolized to an active metabolite in order to exert its pharmacological effect. After absorbtion, $85 \%$ of the drug is broken down by blood esterases, while only $15 \%$ reaches liver. Formation of active metabolite involves twostep process, regulated by isoforms of hepatic cytochrome 450 . Active metabolite then irreversibly binds to $\mathrm{P}_{2} \mathrm{Y}_{12}$ receptor, which prevents ADP binding and subsequent thrombocyte activation.

Variable levels of active metabolite generation are considered the key concept behind altered response to clopidogrel [5].

\section{Variability in clopidogrel response}

Despite high effectivity, thromboembolic events occur even when patients are administered antiplatelet therapy. Interindividual variability in clopidogrel response is considered to be large. Even when data are not accurate, and threshold values vary, studies conclude that poor responders represent between 10 to $40 \%$ of patients [6,7], while coronary stent thrombosis occurred in 5-10\%. Thienopyridine resistance results in inadequate platelet 
inhibition and high on-treatment platelet reactivity. Therefore, low responding patients are exposed to risk of arterial thrombosis and other major adverse cardiovascular events (MACEs) [6]. High on-treatment platelet reactivity is also an established risk factor for occurrence of subacute intrastent thrombosis in patients who underwent percutaneous coronary intervention (PCI). Prospective study of Buonamici et al. [8] monitored patients $(n=804)$ who uderwent coronary stent implantation. Results show that patients with impaired response to clopidogrel were exposed to 3 -fold higher risk of stent thrombosis and cardiac death.

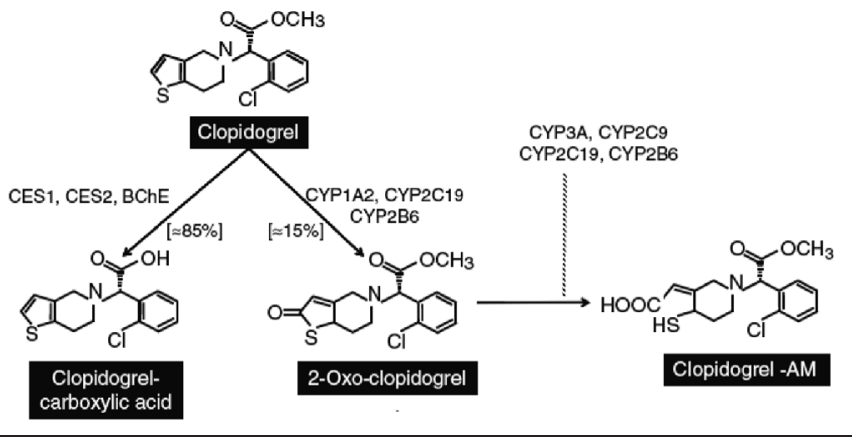

Fig. 2. Clopidogrel metabolism and its conversion to an active metabolite (Clopidogrel- AM) via cytochrome system (CYP). Appx. 85\% of absorbed clopidogrel is broken down by blood esterases (carboxylesterase,CES; butyrylcholinesterase,BChE). (Adapted from : Cattaneo M., Response variability to clopidogrel: is tailored treatment, based on laboratory testing, the right solution? J Thromb Haemost 2012; 10: 327-36.)

Genetic basis of clopidogrel resistance is considered among the primal mechanisms. It can be either caused by polymorphism in genes encoding enzymes of cytochrome system or genetic defects resulting in limited intestinal absorption of prodrug (genes encoding efflux pump P-glycoprotein). Polymorphism in platelet surface receptors is also thought to be one of the inherited mechanisms. Part of variability also depends on clinical patient characteristics, and/or results from interaction with drugs such as some of the proton pump inhibitors (omeprazole), lipophilic statins (i.e. atorvastatin) and calcium channel blockers. Needless to say, non-compliance is considered a frequent cause of poor response to clopidogrel (according to studies these numbers may be as high as $20 \%$ ). Other variables associated with decreased response include advanced age, high body mass index, diabetes mellitus and renal insufficiency in diabetes mellitus. On the other hand, tobacco smoking is associated with increased response via stimulation of CYP1A2 activity [5].

\section{VASP-P ASSAY AND MONITORING RESPONSE TO CLOPIDOGREL}

Reasons stated above, raised the necessity for implementing reliable laboratory test in order to identify the vast group of poor responding patients. While multiple electrode aggregometry (MEA) represents the golden standard for platelet function testing, it does not tar- 
get primarily P2Y12 linked cascade. Paradoxically, despite being referred to as „golden standard" , another major pitfall is standardising the results between multiple laboratories. In recent years, VASP-P flow cytometric assay is emerging into the spotlight as the most promising and most sensitive method. Along with high sensitivity, assay requires only minimal manipulation with sample [9].

Vasodilator Stimulated Phosphoprotein (VASP) is an intracellular platelet protein which is non phosphorylated at basal state. VASP phosphorylation is regulated by the cAMP (cyclic Adenosine Monophosphate) cascade. PGE1 (Prostaglandin E1) activates this cascade (1) whereas it is inhibited by ADP (Adenosine Diphosphate) through P2Y12 receptors. In the test conditions, VASP phosphorylation correlates with the P2Y12 receptor inhibition, whereas its non-phosphorylation state correlates with the active form of P2Y12. The effect of thienopyridines (3) can be demonstrated with PLT VASP/P2Y12 by the persistence of VASP phosphorylation induced by PGEl even with the simultaneous addition of ADP. According to the publication from Aleil B. et al PLT VASP/P2Y12 test is strongly correlated with the inhibition of ADP-induced platelet aggregation due to in vitro specific P2Y12 blockade $(\mathrm{r}=0.72 ; \mathrm{p}<0.0001)[10]$.

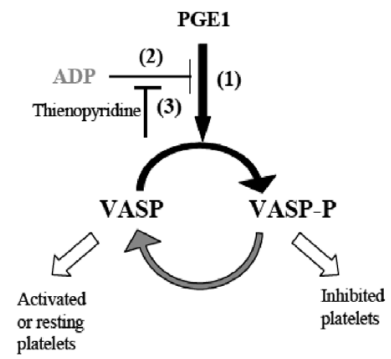

Fig. 3. VASP phosphorylation and ADP mediated platelet activation. (adapted from : http://www.american-diagnostica.de/fileadmin/user_upload/Biocytex/7014-english.pdf) [11].

Method and result interpretation : Sample of citrate blood is incubated with PGE1 and PGE1+ADP. After a cellular permeabilization, VASP under its phosphorylated state is labeled by indirect no wash immunofluorescence using a specific monoclonal antibody(fluorescein izotiocyanate, 16C2, FITC). Dual color flow cytometry analysis allows to compare the two tested conditions and to evaluate for each sample the capacity of ADP to inhibit VASP phosphorylation. In the final step, platelet reactivity index (PRI) is calculated using corrected mean VASP fluorescence intensities (MFIc) in the presence of PGE1 alone (resting platelets) or PGE1 + ADP simultaneously (activated platelets) [9]. PRI represents the ratio of activated/ resting platelets, and is calculated according to the following equation :

$$
\mathrm{PRI}=\left[\left(\mathrm{MFIC}^{\mathrm{PGE} 1}-\mathrm{MFIc}^{(\mathrm{PGE} 1+\mathrm{ADP})}\right) / \mathrm{MFIc}{ }^{\mathrm{PGE} 1}\right] \times 100
$$

The result is PRI value representing index of platelet reactivity to ADP in range of $0-100 \%$. PRI values above $50 \%$ are considered inadequate response to treatment and signal expo- 
sure to increased risk of myocardial infarction, post-PCI stent thrombosis and cardiac death. In patients undergoing coronary stenting the high on-treatment platelet reactivity was associated with higher incidence of major cardiac adverse effects (MACEs) and periprocedural myonecrosis [6].

B.Aleil et al [10] monitored PRI values in group of healthy donors $(n=47)$ compared to the group of patients with ischemic heart disease on clopidogrel antiplatelet treatment $(n=33)$. PRI values of donors were $78.3 \pm 4.6 \%$ compared to $61.1 \pm 17.0 \%$ in clopidogrel receiving group. The PRI of patients treated with clopidogrel for more than 1 week, displayed wide distribution range with values varying from $6.6 \%$ to $85.8 \%$. Moreover, about one-third of the patients treated with clopidogrel had a platelet reactivity equivalent to values in healthy donors or patients not receiving clopidogrel, indicating that desired decrease in platelet reactivity was not attained $[10,11,12]$. The importance of VASP-P assay was also confirmed by Bonello et al who found that adjusting clopidogrel loading dose according to PRI resulted in lower incidence of post-PCI stent thrombosis without the concomitant risk of bleeding [12].

\section{CONCLUSIONS}

Several studies report that further laboratory tests revealed suboptimal response in 15 to $40 \%$ of patients receiving clopidogrel treatment. High on-treatment platelet reactivity is considered a risk factor for occurrence of major cardiovascular events (MACEs) and subacute stent thrombosis. On the contrary, hyperresponders are expected to be in risk of excessive bleeding.

VASP-P flow cytometric assay is emerging as the most promising and most sensitive method to detect patients with suboptimal response. Method is strongly correlated with the inhibition of ADP-induced platelet aggregation due to in vitro specific P2Y12 blockade ( $\mathrm{r}=$ $0.72 ; \mathrm{p}<0.0001)$. In comparison with light transmission aggregometry, VASP-P assay is more valuable for monitoring treatment with $\mathrm{ADP}$ inhibitors since it reflects specifically activity of the P2Y12 receptor. VASP-P assay also gives stable results for more than $24 \mathrm{~h}$ after blood sampling (in contrast to $2 \mathrm{~h}$ for LTA). Results show that more than $33 \%$ of patients are not protected by clopidogrel treatment. However, challenge remains in standardising in vitro results and proposing benchmarks to predict actual clinical outcome, and most importantly, to guide the effective tailored treatment with individual approach.

\section{REFERENCES}

1. O'Connor S, Montalescot G, Collet JP. The P2Y12 receptor as a target of antithrombotic drugs. Purinergic Signalling 2011; 7: 325-332.

2. Cattaneo M. , Aspirin and Clopidogrel : Efficacy, Safety, and the Issue of Drug ResistanceArteriosclerosis, Thrombosis, and Vascular Biology. 2004; 24: 1980-1987)

3. Montalescot G, Wiviott SD, Braunwald E, et al for the TRITON-TIMI 38 investigators. Prasugrel compared with clopidogrel in patients undergoing percutaneous coronary intervention for ST-elevation myocardial infarction (TRITON-TIMI 38): double-blind, randomised controlled trial. Lancet 2009; 373: 723-731

4. Bonaca MP, Steg PG, Feldman L, Canales JF, Ferguson J, Wallentin L, Califf RM, et al. Antithrombotics in Acute Coronary Syndromes. J Am Coll Cardiol 2009; 54, 11: 969-984.

5. Cattaneo M. Response variability to clopidogrel: is tailored treatment, based on laboratory testing, the right solution? J Thromb Haemost 2012; 10: 327-36.

6. Wang X, Zhang D, Zhuang S, Lai Y. Modifying Clopidogrel Maintenance Doses According to VasodilatorStimulated Phosphoprotein Phosphorylation Index Improves Clinical Outcome in PatientsWith Clopidogrel Resistance. Clin Cardiol 2011; 34, 5, 332-338.

7. Wallentin L, et al. P2Y12 inhibitors : differences in properties and mechanisms of action and potential consequences for clinical use. European Heart Journal 2009; 30, 1964-1977. 
8. Buonamici P, Marcucci R, Migliorini A, et al. Impact of platelet reactivity after clopidogrel administration on drug-eluting stent thrombosis. J Am Coll Cardiol 2007; 49: 2312-7.

9. Gachet C, B.Aleil B, Testing antiplatelet therapy. European Heart Journal Supplements 2008; 10 (Suppl A), A28-A34.

10. Aleil B, Ravanat C, Cazenave JP, Rochoux G, Heitz A, Gachet C. Flow cytometric analysis of intraplatelet VASP phosphorylation for the detection of clopidogrel resistance in patients with ischemic cardiovascular diseases. J Thromb Haemost 2005; 3: 85-92.

11. STAGO - Biocytex ${ }^{\circledR}$ (France), Manual for PLT VASP/P2Y12 kit; http://www.american-diagnostica.de/fileadmin/user_upload/Biocytex/7014-english.pdf

12. Bonello L, Camoin-Jau L, Armero S, et al. Tailored clopidogrel loading dose according to platelet reactivity monitoring to prevent acute and subacute stent thrombosis. Am J Cardiol. 2009;103:5-10.

\section{Acknowlegements:}

This work was supported by project „Center of Excellence for Perinatology Research“ CEPVII (ITMS 26220120036) and academic grants of Comenius University.

Received: March, 20, 2013

Accepted: April, 3, 2013 